\title{
The Tor Vergata Synoptic Solar Telescope (TSST): A robotic, compact facility for solar full disk imaging
}

\author{
Luca Giovannelli $^{1}$, Francesco Berrilli ${ }^{1}{ }^{*}$, Daniele Calchetti ${ }^{1}$, Dario Del Moro ${ }^{1}$, Giorgio Viavattene ${ }^{1,2}$, \\ Ermanno Pietropaolo ${ }^{3}$, Marco Iarlori ${ }^{4}$, Vincenzo Rizi ${ }^{4}$, Stuart Mark Jefferies ${ }^{5}$, Maurizio Oliviero ${ }^{6}$, \\ Luciano Terranegra $^{6}$, and Neil Murphy ${ }^{7}$
}

${ }^{1}$ Department of Physics, University of Rome Tor Vergata, 00133 Roma, Italy

2 INAF - Astronomical Observatory of Rome, 00078 Roma, Italy

3 INFN-GSGC L'Aquila and DSFC University of L'Aquila, 67100 L'Aquila, Italy

${ }^{4}$ INFN-GSGC L'Aquila and CETEMPS-DSFC University of L'Aquila, 67100 L'Aquila, Italy

${ }^{5}$ Department of Physics and Astronomy, Georgia State University, Atlanta, 30303 GA, USA

${ }^{6}$ INAF - Capodimonte Astronomical Observatory, 80131 Napoli, Italy

7 Jet Propulsion Laboratory - NASA, Pasadena, 91109 CA, USA

Received 15 June 2020 / Accepted 12 October 2020

\begin{abstract}
By the continuous multi-line observation of the solar atmosphere, it is possible to infer the magnetic and dynamical status of the Sun. This activity is essential to identify the possible precursors of space weather events, such as flare or coronal mass ejections. We describe the design and assembly of TSST (Tor Vergata Synoptic Solar Telescope), a robotic synoptic telescope currently composed of two main full-disk instruments, a $\mathrm{H} \alpha$ telescope and a Potassium (KI D1) magneto-optical filter (MOF)based telescope operating at $769.9 \mathrm{~nm}$. TSST is designed to be later upgraded with a second MOF channel. This paper describes the TSST concepts and presents the first light observation carried out in February 2020. We show that TSST is a low-cost robotic facility able to achieve the necessary data for the study of precursors of space weather events (using the magnetic and velocity maps by the MOF telescope) and fast flare detection (by the $\mathrm{H} \alpha$ telescope) to support Space Weather investigation and services.
\end{abstract}

Keywords: solar physics / instrumentation / solar telescope / synoptic telescope / ground telescope / network

\section{Introduction}

Ground-based networks of solar synoptic telescopes are a powerful tool for characterizing the activity status of our star and detecting/forecasting sources of Space Weather events. The use of a network of fully-robotic compact synoptic telescopes represents a low-cost and high-efficiency support to Space Weather (SWe) services and a valuable complement to solar space missions, e.g., Solar Orbiter or Parker Solar Probe, and context imaging for next-generation 4-m class groundbased solar telescopes such as DKIST and EST (e.g. Jurčák et al., 2019; Rast et al., 2019).

The Tor Vergata Synoptic Solar Telescope (TSST) is a compact and robotic multi-channel instrument designed to be compliant to the main objectives described in the framework of the SPRING project, developed under the H2020-SOLARNET program funded by the European Union, and focused on a preliminary design study of a synoptic solar observing facility

\footnotetext{
*Corresponding author: francesco. berrilli@roma2. infn. it
}

(Gosain et al., 2018). Indeed, as reported in Gosain et al. (2018) we know that to better understand the violent solar phenomena which represent the main sources of SWe events, such as flares or coronal mass ejections (CMEs), we need multi-line synoptic observations, e.g., multi-height velocity and magnetic maps, to enable tomographic inference of solar physical parameters. The observation of different layers of the solar atmosphere is in fact necessary to follow the dynamics and the evolution of these inherently 3D phenomena. Currently, solar synoptic observations are carried out on space, as an example by the suite of instrument on board the Solar Dynamics Observatory (SDO) (Pesnell et al., 2012), or by networks of ground-based telescopes distributed in longitude, as the Global Oscillation Network Group (Harvey et al., 1996) and the Birmingham Solar Oscillations Network (BiSON) (Chaplin et al., 1996), or from high latitude observatories, e.g., from the South Pole during the austral summer (Jefferies et al., 1988).

In the last years, the guidelines for new synoptic solar observing networks have been proposed to update the technology and objectives of the networks created in the $90 \mathrm{~s}$ 
(e.g. Hill et al., 2013; Elsworth et al., 2015). The proposed guidelines provide an observational framework for conducting strategic planning in solar science focused on SWe. In more detail, they include multi-height long term monitoring of velocity and solar magnetic maps.

Multi-height long term velocity maps, i.e. Dopplergrams, allow us to investigate the dynamics of the solar atmosphere and of subsurface flows via signal analysis and helioseismology. In connection with SWe issues, these maps allow us to study global solar dynamo and its relation with the solar cycle and magnetic field emergence and cancellation in quiet and active Sun.

Multi-height long term solar magnetic field maps, allow us to understand solar global dynamo and solar activity cycles and surface flows via feature tracking (e.g. Giannattasio et al., 2014, 2019).

MOF-based systems proved to play a major role in exploiting the magnetic signal of the Sun, especially in combination with the possibility to obtain from the same instrument intensity and velocity signals altogether. For instance, in Moretti et al. (2001) a first full disk $l-v$ analysis was performed from a MOF dataset, together with a local analysis, exploring the intensity-velocity phase relation and enabling to investigate the source of solar oscillations. Likewise, in Moretti et al. (2003), the magnetic-field-velocity phase difference has been computed, finding evidence of global magnetic oscillations.

The combined information of dynamic evolution of the solar atmosphere and magnetic field allows us to shed light on the physical processes underlying the rapid transients, like flares and CMEs, generated by solar activity, making possible and reliable the forecasting of these potentially destructive events due to their impact on technological infrastructures in space and ground-based.

\section{Telescope concept}

TSST consists of a dual channel solar telescope composed of an $\mathrm{H} \alpha$ channel, operating at $656.28 \mathrm{~nm}$, and a potassium MOF-based channel, operating at $769.9 \mathrm{~nm}$ and sounding an atmospheric layer about $300-400 \mathrm{~km}$ over the solar photosphere. Both channels are mounted on a Sky-Watcher EQ8 PRO Skynscan equatorial platform (load capacity of $50 \mathrm{~kg}$ ). One purpose in assembling TSST was to exploit as many offthe-shelf optical and mechanical components as possible to speed-up the development and contain the final cost (Cacciani et al., 1995). The overall budget need for the TSST is $\sim 50 \mathrm{k€}$, while, for instance, instruments based on Fabry-Pérot interferometers with similar magnetic performance require $\sim 300 \mathrm{k} €$ just for the etalons and controllers components (see e.g. Berrilli et al. 2010, 2015). As far as robotization and remote control of the telescope are concerned, we collaborated with highly experienced groups, and we have purchased components, telescope mount and dome controlled by off-the-shelf software packages. The TSST will be one node of a network of solar observatories, based on the MOF technology, together with the VAMOS telescope in Naples (Oliviero et al., 1998) and the MOTH instrument (Cacciani et al., 2003) managed by the Georgia State University (GSU) team and used to simultaneously map the line-of-sight (LoS) velocity at two heights in the solar atmosphere and able to determine the travel time, i.e., the propagation speed of sound waves, in the solar atmosphere (Jefferies et al., 2019).

A dedicated computer is used to control the robotic dome and mount, and acquire images using CMOS cameras. The current TSST CMOS detectors, provided by the Remote Sensing for Space Sciences Research Group at the Department of Physics and Astronomy of GSU, are Dalsa Pantera 1M60 cameras ${ }^{1}$ operating at room temperature without cooling. This camera uses the FTT 1010-M monochrome progressive-scan frametransfer image sensor with $1024 \times 1024$ pixels of $12 \mu \mathrm{m}$ and has a peak quantum efficiency of $45 \%$ at $500 \mathrm{~nm}$ (about $25 \%$ at $656.28 \mathrm{~nm}$ and $12 \%$ at $769.9 \mathrm{~nm}$ ). The Pantera 1M60 uses a Frame Grabber RoHS Xcelera-CL PX4 PCI card and cable to control/acquire images.

The TSST acquires simultaneously photospheric LoS velocity and magnetic maps (MOF channel) and chromospheric images ( $\mathrm{H} \alpha$ channel). The KI D1 MOF channel and the $\mathrm{H} \alpha$ channel sound two layers of the solar atmosphere about $350 \mathrm{~km}$ (Tomczyk et al., 1995) and $1500 \mathrm{~km}$ (Vernazza et al., 1981; Leenaarts et al., 2012) over the solar photosphere, respectively. The maximum available cadence for magnetograms and Dopplergrams from the MOF channel is $15 \mathrm{~s}$. The $\mathrm{H} \alpha$ channel will have a cadence of $5 \mathrm{~s}$ for real time flare detection, localization, and intensity estimate (Piazzesi et al., 2012). The database will provide hourly magnetograms, dopplergrams and $H \alpha$ images, together with higher cadence datasets during flare events.

The TSST is a telescope designed to accommodate a third MOF channel hosting: Sodium D2 line cells operating at $589 \mathrm{~nm}$ and sounding an atmospheric layer about $650 \mathrm{~km}$ over the solar photosphere (Finsterle et al., 2004a), or Calcium I cells operating at $422 \mathrm{~nm}$ and sounding an atmospheric layer about $1000 \mathrm{~km}$ over the solar photosphere (Murphy et al., 2005), or Helium I cells operating at $1083 \mathrm{~nm}$ and sounding an atmospheric layer about $1900 \mathrm{~km}$ over the solar photosphere (Murphy et al., 2005). A Sodium MOF channel would allow a direct comparison with the equivalent channel of the MOTH instrument, reinforcing the network capabilities. Therefore, the preferred choice at the moment is a third channel based on Sodium MOF cells.

\subsection{The $\mathrm{H} \alpha$ channel}

The H $\alpha$ channel of the TSST is a Daystar SR-127 QT, an achromatic refractor with $127 \mathrm{~mm}$ aperture and a focal length of $2667 \mathrm{~mm}$. The telescope features a narrow band filter with a passband of $0.04 \mathrm{~nm}$ centered at the $\mathrm{H} \alpha$ line. The filter is a temperature stabilized etalon in collimated configuration, and it is the same filter used in the GONG network for the $\mathrm{H} \alpha$ channel to monitor the chromospheric transients (Giersch, 2013). The $\mathrm{H} \alpha$ channel is equipped with a focal reducer, acting also as a field curvature corrector, to match the Dalsa Pantera 1M60 pixel scale of the MOF channel (2.6 arcsec/pixel).

The main goal of this TSST channel is the real time detection of flaring region (Piazzesi et al., 2012; Pötzi et al., 2015, 2018) during the impulsive and flash phases when the intensity reaches its maximum (Benz, 2017). $\mathrm{H} \alpha$ images are extremely

\footnotetext{
$\overline{{ }^{1} \mathrm{http}: / / \text { teledynedalsa.com/ }}$
} 
important for ground based SWe monitoring (see, e.g. Veronig \& Pötzi, 2016) and to automatically detect and track structures in the solar chromosphere, as filaments (Bernasconi et al., 2005; Fuller et al., 2005; Bonnin et al., 2013) and prominences (Wang et al., 2010), that can be associated with flare and coronal mass ejections. We intend to use the information about real-time flare detection and chromospheric structure position and evolution from the TSST, together with photospheric magnetic field provided by the MOF channel, to improve forecasting algorithms for SWe events already running for the SWERTO service (see more details in Sect. 3).

\subsection{The Potassium MOF channel}

The Potassium MOF channel telescope has been customdesigned with Zemax ${ }^{\circledR}$ software. The optical and polarimetric scheme, discussed in detail in Viavattene et al. (2020), is reported in Figure 1. It is a double-keplerian telescope with an imaging lens and a corrective lens, and two folding mirrors (M1 and M2), used to reduce the linear dimensions of the instrument. Solar light, passing through the objective lens L1, is filtered by an IR filter (hot mirror), a UV filter and a Potassium K filter. L2, L3, L4 and L5 are identical doublets. In the collimated beam between L2 and L3, an interference filter (IF) with a passband of $1.7 \mathrm{~nm}$ centered at $770 \mathrm{~nm}$, a quarterwave plate (QWP), an half-wave plate (HWP), which are the magnetic modulator, and the MOF cell between two crossed linear polarizers (P1 and P2) are placed. In the collimated beam between L4 and L5, a linear polarizer P3, a second HWP, a second QWP and the wing selector (WS) cell are placed (Doppler modulator). Finally, the L5 combined with the corrective lens (CL) forms an aberration-free image on the CCD (Viavattene et al., 2020). The polarimetric scheme is similar to that of the VAMOS telescope. The QWPs, the rotating HWPs and the linear polarizers are used in combination in order to select the right and left circular polarization of the red and blue wings that are used to compute the Dopplergrams and magnetograms.

The core technology of TSST is the magneto-optical filter (MOF). The MOF is a cell that enables resonant optical spectroscopy in alkali metal vapours, in our case potassium, with a strong longitudinal magnetic field inside. These types of cells have a wide range of applications, but in the field of solar physics they were introduced in the 70 s by the solar physics group of the Astronomical Observatory of Rome (Agnelli et al., 1975; Cacciani \& Fofi, 1978) and subsequently developed for ground-based (e.g., synoptic telescopes or Antarctic campaigns) or space instruments (Berrilli et al., 1987; Cacciani et al., 1997; Oliviero et al., 1998; Finsterle et al., 2004b; Haberreiter et al., 2007; Berrilli et al., 2010; Moretti et al., 2010; Ambrož \& Pötzi, 2013).

A MOF-based telescope is typically divided into three sections:

- Magnetic modulator, which separates right $\left(\sigma^{+}\right)$and left circular $\left(\sigma^{-}\right)$polarization states produced by solar LoS magnetic field.

- MOF section, which produces a passband with peaks in the red $(R)$ and blue wings $(B)$ of selected absorption line.

- Doppler modulator which absorbs one of the peaks and let the other pass.
After the Doppler modulator, the acquisition of both polarization states in the $R$ and $B$ wings $\left(R^{+}, R^{-}, B^{+}\right.$and $\left.B^{-}\right)$ allows the estimate of the LoS velocity, i.e., Dopplergrams, $(D)$ and LoS magnetic field $(M)$ of the Sun (e.g. Calchetti et al., 2020):

$$
\begin{gathered}
D=\frac{R^{+}-B^{+}}{R^{+}+B^{+}}+\frac{R^{-}-B^{-}}{R^{-}+B^{-}} \\
M=\frac{R^{+}-B^{+}}{R^{+}+B^{+}}-\frac{R^{-}-B^{-}}{R^{-}+B^{-}} .
\end{gathered}
$$

A telescope based on this technology can be assembled with different configurations and optical designs, but these three sections are mandatory to acquire Dopplergrams and magnetograms of the Sun.

Velocity and magnetic maps obtained with equations (1) and (2) are proportional to the LoS velocity and magnetic field intensity signals. It is possible to calibrate the measured quantities following the procedure described in Cacciani et al. (1997), Oliviero et al. (1998) and Vogt et al. (1999). The VAMOS telescope (Oliviero et al., 2010) and the MOTH telescope (Forte et al., 2018) represents two different schemes that can be used for a MOF-based instrument. The VAMOS telescopes alternates all the images acquired by the instrument on the same camera, whereas the MOTH separates the Doppler signature on two different detectors.

The spectral calibration of the MOF and WS cells is fundamental to obtain the best passband of the instrument and for its proper operation. The magnetic field and the temperature (so the vapour density) are the two physical parameters to be determined for each cell. MOF cells are now controlled by a digital PID temperature controller powered by a relay output. This system can reach the stability of $0.1^{\circ} \mathrm{C}$ for the temperature inside the cell, which ensures high stability in the passband peaks and then in the data acquired by the telescope. The central wavelength of the MOF reference line has no drift because is due to the atomic transition of the potassium vapour inside the cell. This is the reason of the extreme stability of MOF transmission profiles. Given the dependence of the Faraday rotation from the temperature, only a minor shift of the blue and red passbands with respect to the central wavelength is present. From the measured line profiles (Fig. 1), we estimate a shift of about $3 \times 10^{-4} \mathrm{~nm} /{ }^{\circ} \mathrm{C}$, in line with Severino et al. (2007). Given the cells' temperature stability of $0.1^{\circ} \mathrm{C}$, the instrument spectral stability is $3 \times 10^{-5} \mathrm{~nm}$. The holder of the cell is also collected in a thermal controlled box which dissipates the heat outside the box of the telescope. This is mandatory to maintain a longitudinal temperature gradient inside the cell and to avoid the heat up of the whole MOF channel. For further details on the spectral calibration procedure, see Calchetti et al. (2020). We set the magnetic flux density at $1.23 \mathrm{kG}$ and the temperature at $166.5^{\circ} \mathrm{C}$ for the MOF cell, and at $2.26 \mathrm{kG}$ and at $185^{\circ} \mathrm{C}$ for the WS cell, respectively. The red and blue passbands measured at different WS cell temperatures are reported in Figure 2. In this way, the distance between the two peaks of the MOF passband is $20 \times 10^{-3} \mathrm{~nm}$. The transparency of the red and blue peaks are $47.5 \%$ and $49 \%$, respectively, with a FWHM of $5.6 \times 10^{-3} \mathrm{~nm}$. The contamination from the other wing is lower than $10 \%$. 


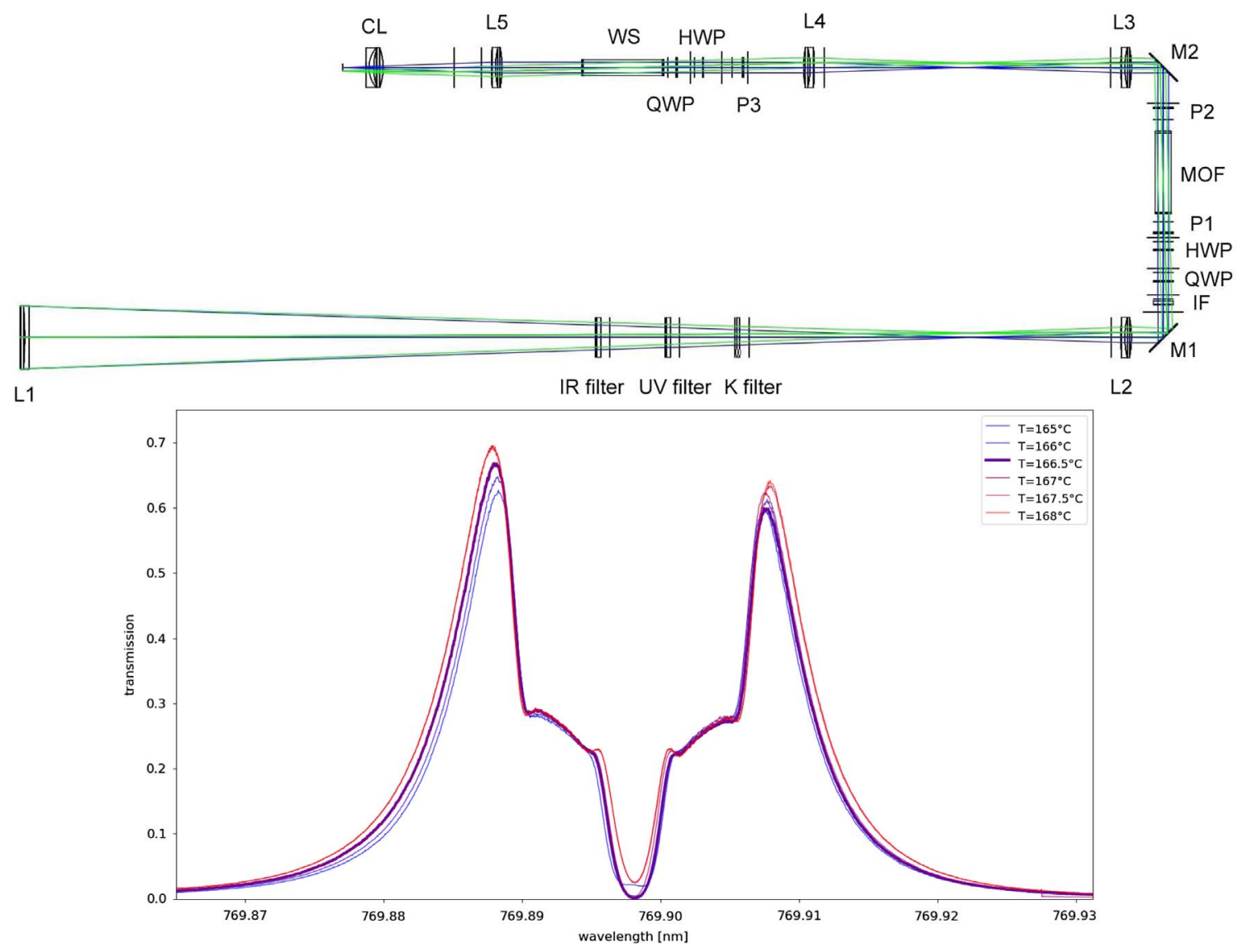

Fig. 1. The TSST potassium channel f/12 optical path. L1 is the $80 \mathrm{~mm}$ objective lens (top). L2, L3, L4 and L5 are achromatic doublet lens. M1 and M2 are flat folding mirrors. QWP and HWP are quarter-wave plates and half-wave plates, respectively. P1, P2 and P3 are linear polarizers. CL is a group of corrective lenses. After the $\mathrm{P} 2$ element the instrument passbands, typical of MOF instruments, is the one shown in the bottom panel. The measured passband is shown at different MOF cell temperatures. Thicker solid line corresponds to the chosen working temperature of the cell. Only one of the two wing passbands is later selected by the WS section, as shown in Figure 2. It is possible to select one of the wing passbands rotating the appropriate retarders.
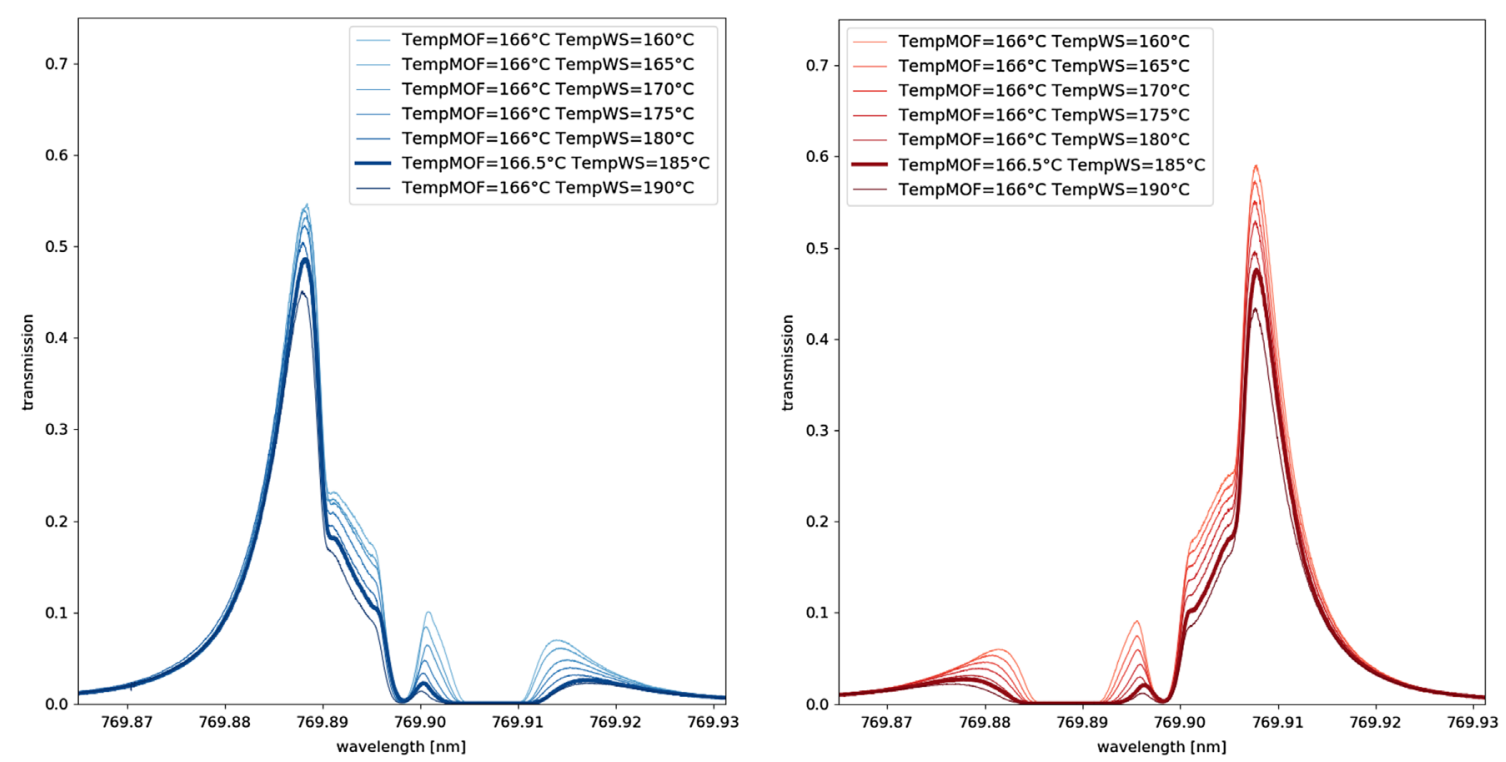

Fig. 2. Left panel: Blue wing passbands at different WS cell temperatures. Right panel: Red wing passbands measured at different WS cell temperatures. The MOF cell temperature is fixed at $166.5^{\circ} \mathrm{C}$. Thicker solid lines correspond to the chosen working temperature of the WS cell. 

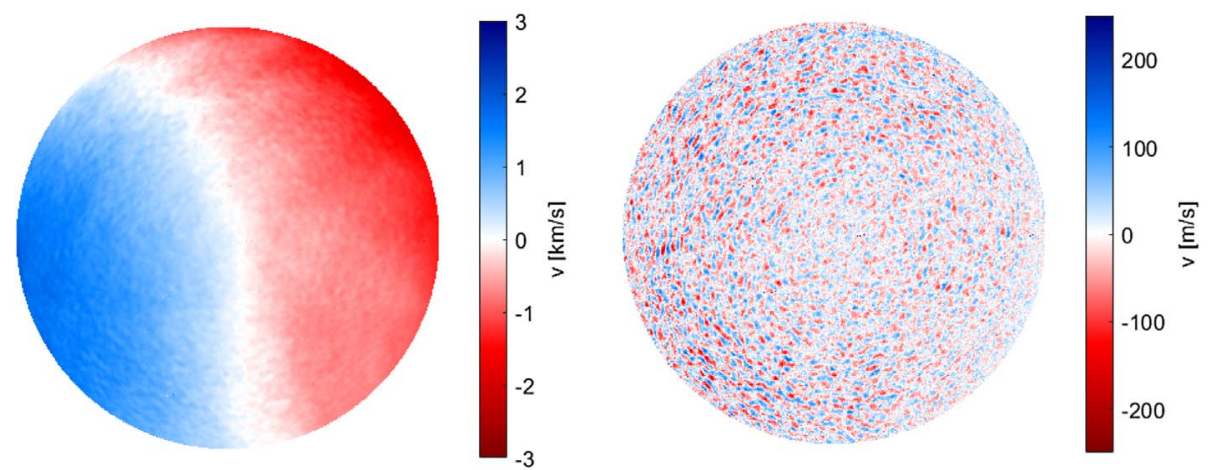

Fig. 3. These dopplergrams of solar disk from TSST MOF channel were acquired on February 7, 2020 and represent: (Left panel) LoS velocity map of the Sun, the Dopplergram is dominated by the Sun's rotation; (Right panel) same LoS velocity map after Sun's rotation removal showing the supergranulation and $p$-modes pattern. On the right of the Dopplergrams the velocity scales are reported.

\subsection{TSST remote control and observatory site}

As far as the remote control and robotization of the system are concerned, we need to consider two separate aspects: on the one hand, there is the control and monitoring of the system that is developed even in the absence of remote installation of the telescope. On the other hand, the possibility of operating remotely and in automatic mode needs the integration of the system with a moving dome. As discussed in the previous sections, a primary requirement is to control the system, i.e., dome, telescope, detectors, observation scheduling and data archiving, with a dedicated software application. The first task is then the realization of an application that integrates all the required functions and works both through a graphic interface and in no-graphic mode. The development of the second part, i.e. robotization and integration with a moving dome, depends on the type of dome used. At the moment, we are planning to use a $3 \mathrm{M}$ dome, manufactured by ScopeDome, whose software is compliant with the ASCOM (AStronomy Common Object Model) platform framework whose drivers for the telescope mount are also available.

In partnership with the University of L'Aquila and Istituto Nazionale di Fisica Nucleare (INFN), and after an agreement with the Instituto de Astrofsica de Canarias (IAC), we plan to install the TSST, after a period of commissioning in Italy, at the Observatorio del Roque de Los Muchachos (ORM) on the Canary island of La Palma, Spain, in the facility of the Raman LIDAR for the pre-production phase of Cherenkov Telescope Array (Iarlori et al., 2019) equipped with a robotic ScopeDome $3 \mathrm{M}$. The ORM is one of the best sites for solar astronomy in the world (e.g. Townson et al., 2015) and has been recently selected as final site for EST. The ORM provides exceptional data bandwidth to continental Europe, enabling data storage in Italy. Furthermore, data transfer can be enhanced using lossless compression techniques (see e.g. Del Moro et al., 2017). Moreover, the ORM offers for the Italian solar community logistical advantages with the presence of INFN and INAF facilities.

\subsection{First light for TSST at Solar station of UNITOV Physics Department}

On 2020 February 7th, we acquired the first light of the TSST MOF channel at Solar and UV station at the Department of Physics of the University of Rome Tor Vergata. The MOF channel of the telescope was in the assembly phase at that time; therefore, a different optical scheme has been used with respect to that shown in the Figure 1. It was mounted on the optical bench of the Solar Physics Laboratory and fed with the solar beam from the heliostat. A Grasshopper GS3-U3-28S5M-C camera, with $1920 \times 1440$ pixels and pixel width $=4.54 \mu \mathrm{m}$, and a proper focal reducer has been used instead of the Dalsa Pantera 1M60, for availability issues.

Intensity, flat field and leakage images in all four bands $\left(R^{+}, R^{-}, B^{+}\right.$and $\left.B^{-}\right)$, necessary for the first level calibration described in Forte et al. $(2018,2020)$, have been acquired during this run. In more detail, final images are the combination of shorter acquisitions $(40 \mathrm{~ms})$ with a full exposure time of $1 \mathrm{~s}$ per passband. The main goal of this first light observation was to confirm the alignment of the optical elements and the excellent outcome of the spectral calibration. During nominal operations, the quality of the observations is affected by the unpolarized signal leakage, due to the non-ideal polarizers which constitute MOFs. Leakage images are mandatory to estimate the signal to noise ratio of the observation and are acquired with MOF cell turned off. With this setup, the light reaching the sensor is limited by the cross polarizers in the MOF section. The intensity of the leakage signal depends on the polarizer extinction factor, proper alignment of the polarizers and all-optical elements. To estimate the quality of the MOF observations we use the ratio between the average signal acquired during nominal operation and the corresponding mean leakage signal for each of the four polarization and spectral states $\left(R^{+}, R^{-}, B^{+}\right.$and $\left.B^{-}\right)$. We find an excellent signal to leakage ratio of $\approx 25$. We expect an increase in this value after the reduction of the stray light. This will be achieved with the finalization of the assembly phase, consisting in the addition of baffles and light traps and the enclosure of the whole optical path. High signal to noise ratio is also the evidence of a proper alignment of all the optical elements performed during the spectral calibration.

The first light dataset was used to compute a full-disk Dopplergram as a test of the required science data products. The Dopplergram showing the LoS velocity map was computed following the standard pipeline for MOF telescopes described in Forte et al. $(2018,2020)$ (Fig. 3). In the left panel, we show the Dopplergram, with a clear signal of the Sun rotation. LoS velocity has been calibrated using the literature rotation signal 


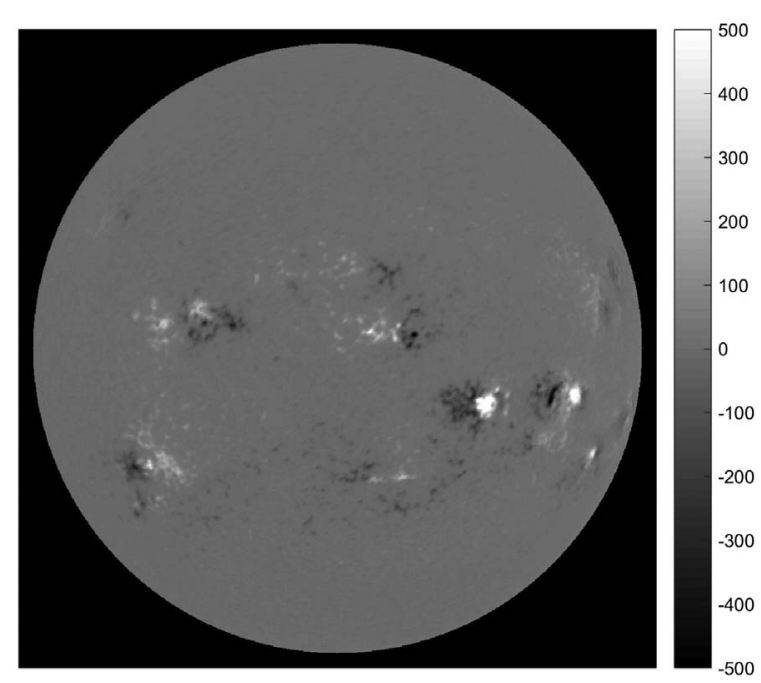

Fig. 4. Sample of a solar LoS magnetic field synoptic map $(M)$ acquired by the $\mathrm{K}$ channel of the MOTH instrument at 19:25 UT 2014 July 9th. The pixel scale of the images is $2 \mathrm{arcsec} /$ pixel, similar to the TSST Potassium MOF channel. The colorbar is in arbitrary units. See Forte et al. (2020) for more information.

value at the Sun limb. Furthermore, the large scale signal of solar rotation was isolated using a gaussian filter. Once subtracted, the Doppler pattern due to e.g. supergranulation and $p$-modes emerges (right panel of Fig. 3). Note the different amplitude of the signal in this latter case.

No active regions were present on the Sun during the the first light observation. In Figure 4 we show, as a reference, a sample of the magnetograms acquired by the MOTH potassium channel during a day of higher solar activity (2014 July 9th). The MOTH instrument shares with the TSST a similar instrumental setup. The TSST is expected to reach similar sensitivities for the LoS velocity and magnetic fields with respect to the MOTH instrument. For the MOTH dataset taken in consideration here, with $5 \mathrm{~s}$ integration time, the achievable sensitivites are, respectively, $7 \mathrm{~m} / \mathrm{s}$ and $5 \mathrm{G}$ (see Forte et al. 2020).

\section{Future perspectives and conclusion}

The University of Rome Tor Vergata is currently running a nowcasting and forecasting service, SWERTO (Berrilli et al., 2018, 2019a), developed for Space Weather awareness and risk control at regional level in Italy. At present, it uses GONG $\mathrm{H} \alpha$ images and SDO/HMI magnetograms among the many input data. However, TSST data products are foreseen as an additional input to the SWERTO system. TSST Magnetograms can be used to estimate the flare probability of active regions using the $R$ value, i.e. the total unsigned magnetic flux introduced by Schrijver (2007) and currently implemented in SWERTO using SDO/HMI data, and novel flare forecasting parameters currently under evaluation. $\mathrm{H} \alpha$ emissions can trigger flare nowcasting procedures and, together with the detection of chromospheric structures (prominences and filaments) position can be used to estimate the coronal mass ejections (CMEs) starting sites. This allows to compute CMEs propagation direction, helping mitigating projection effects in the forecast of interplanetary coronal mass ejections (ICMEs) (see, e.g. Del Moro et al., 2019). SWERTO is currently running an ICMEs forecasting tool based on the probability drag based model, that can provide a fast and accurate forecast of ICMEs arrival time at Earth (Napoletano et al., 2018; Berrilli et al., 2019b).

At the national level, the Italian Space Agency (ASI) has recently started an effort to coordinate the several Space Weather activities currently running in the universities, research institutes and industries (Plainaki et al., 2020). ASI proposed the development of a national scientific Space Weather data center, the ASI SPace weather InfraStructure (ASPIS), to collect data from the different Italian Space Weather assets and provide the information to the stakeholders. One of the crucial aspects of ASPIS is the optimization of the observational coverage, providing an organic view of the Space Weather events through the combination of ground-based and space observations. The TSST in this context is one of the foreseen key ground-based resources, especially if integrated into a network of similar synoptic telescopes such as the VAMOS telescope in Naples (Oliviero et al., 1998) and the MOTH instrument (Cacciani et al., 2003) managed by Georgia State University, being able to provide magnetograms to the community independently from the availability of space observations.

In conclusion, the TSST is a dual channel telescope, with capabilities to provide LoS velocity maps and magnetograms from the solar atmospheric layer at 300-400 km above the photosphere (potassium KI D1 line) and intensity maps from the $\mathrm{H} \alpha$ line. The spatial resolution is 2.6 arcsec/pixel for both channels and the cadence is up to $15 \mathrm{~s}$ for the MOF channel and $5 \mathrm{~s}$ for the $\mathrm{H} \alpha$ channel respectively. The modular design of the telescope foresees additional channels in the future, to augment diagnostic capabilities providing magnetograms and Dopplergrams from different layers in the solar atmosphere. TSST data can be exploited together with complementary information provided by other instruments, such as MOTH telescope, GONG network and SDO/HMI, to reconstruct 3D magnetic configuration of the solar atmosphere, providing new tools for Space Weather nowcasting and forecasting capabilities.

Acknowledgements. SWERTO has been financed by the Regione Lazio FILAS-RU-2014-1028 grant (November 2015 - October 2017). This research is partially supported by the Italian MIUR-PRIN grant 2017APKP7T on Circumterrestrial Environment: Impact of Sun-Earth Interaction. SMJ was funded by award 1829258 from the National Science Foundation. The authors thank the anonymous reviewers for their valuable help in improving the manuscript. The editor thanks Sanjay Gosain and an anonymous reviewer for their assistance in evaluating this paper.

\section{References}

Agnelli G, Cacciani A, Fofi M. 1975. The magneto-optical filter. I: Premilinary observations in Na D lines. Sol Phys 44(2): 509-518. https://doi.org/10.1007/BF00153229.

Ambrož P, Pötzi W. 2013. Horizontal Velocities in Solar Filament Channel. Cent Eur Astrophys Bull 37: 495-500.

Benz AO. 2017. Flare observations. Living Rev Sol Phys 14(1): 2. https://doi.org/10.1007/s41116-016-0004-3. 
Bernasconi PN, Rust DM, Hakim D. 2005. Advanced automated solar filament detection and characterization code: Description, performance, and results. Sol Phys 228(1-2): 97-117. https://doi. org/10.1007/s11207-005-2766-y.

Berrilli F, Bruno R, Egidi FA. 1987. Preliminary study of a prototype flight instrument for the detection of Doppler images of the Sun. Nota interna Dipartimento di Fisica II Università di Roma. ROM2F/035/87. URL https://art.torvergata.it/retrieve/handle/ 2108/254187/501800/berrilli1987.pdf.

Berrilli F, Bigazzi A, Roselli L, Sabatini P, Velli M, et al. 2010. The ADAHELI solar mission: Investigating the structure of Sun's lower atmosphere. Adv Space Res 45(10): 1191-1202. https://doi. org/10.1016/j.asr.2010.01.026.

Berrilli F, Soffitta P, Velli M, Sabatini P, Bigazzi A, et al. 2015. ADAHELI: exploring the fast, dynamic Sun in the X-ray, optical, and near-infrared. J Astron Telesc Instrum Syst 1(4): 044006. https://doi.org/10.1117/1.JATIS.1.4.044006.

Berrilli F, Casolino M, Del Moro D, Forte R, Giovannelli L, et al. 2018. SWERTO: A regional space weather service. In: Space weather of the heliosphere: Processes and forecasts, Foullon C, Malandraki OE (Eds.), Vol. 335 of IAU Symposium, pp. 348-351. https://doi.org/10.1017/S1743921318000054.

Berrilli F, Casolino M, Cristaldi A, Del Moro D, Forte R, et al. 2019a. Introducing SWERTO: A regional space weather service. Nuovo Cimento C Geophys Space Phys C 42(1): 47. https://doi. org/10.1393/ncc/i2019-19047-4.

Berrilli F, Cristaldi A, Del Moro D, Forte R, Giovannelli L, Napoletano G, Pietropaolo E. 2019b. The probabilistic drag based model for ICME propagation. Nuovo Cimento C Geophys Space Phys C 42(1): 39. https://doi.org/10.1393/ncc/i2019-19039-4.

Bonnin X, Aboudarham J, Fuller N, Csillaghy A, Bentley R. 2013. Automation of the filament tracking in the framework of the HELIO project. Sol Phys 283(1): 49-66. https://doi.org/10.1007/ s11207-012-9985-9.

Cacciani A, Fofi M. 1978. The magneto-optical filter. II. Velocity field measurements. Sol Phys 59(1): 179-189. https://doi.org/ 10.1007/BF00154941.

Cacciani A, Moretti PF, Smith EJ. 1995. Evaluation of a Portable and Inexpensive MOF Unit for Doppler Imaging. In: GONG 1994. Helio- and Astro-Seismology from the Earth and Space, Ulrich RK, Rhodes EJ, Dappen W (Eds.), Vol. 76 of Astronomical Society of the Pacific Conference Series, $440 \mathrm{p}$.

Cacciani A, Moretti PF, Rodgers WE. 1997. Measuring doppler and magnetic fields simultaneously. Sol Phys 174: 115-128. https://doi.org/10.1023/A:1004935524038.

Cacciani A, Jefferies SM, Finsterle W, Rapex P, Knox A, Giebink C, di Martino V. 2003. A new instrument for sounding the solar atmosphere. In: GONG+2002. Local and global helioseismology: The present and future, Sawaya-Lacoste H (Ed.), Vol. 517 of ESA Special Publication, pp. 243-245.

Calchetti D, Viavattene G, Berrilli F, Del Moro D, Giovannelli L, Oliviero M. 2020. Tor vergata synoptic solar telescope: Preliminary optical design and spectral characterization. In: Journal of Physics Conference Series, Vol. 1548 of Journal of Physics Conference Series, IOP Publishing, 012005 p. https://doi.org/ 10.1088/1742-6596/1548/1/012005.

Chaplin WJ, Elsworth Y, Howe R, Isaak GR, McLeod CP, Miller BA, van der Raay HB, Wheeler SJ, New R. 1996. BiSON performance. Sol Phys 168(1): 1-18. https://doi.org/10.1007/BF00145821.

Del Moro D, Giovannelli L, Pietropaolo E, Berrilli F. 2017. JP3D compression of solar data-cubes: Photospheric imaging and spectropolarimetry. Exp Astron 43(1): 23-37. https://doi.org/ 10.1007/s10686-016-9518-X.
Del Moro D, Napoletano G, Forte R, Giovannelli L, Pietropaolo E, Berrilli F. 2019. Forecasting the 2018 February 12th CME propagation with the P-DBM model: A fast warning procedure. Ann Geophys 62(4): 456.

Elsworth Y, Broomhall A-M, Gosain S, Roth M, Jefferies SM, Hill F. 2015. The importance of long-term synoptic observations and data sets for Solar Physics and Helioseismology. Space Sci Rev 196(1-4): 137-166. https://doi.org/10.1007/s11214-015-0212-5.

Finsterle W, Jefferies SM, Cacciani A, Rapex P, McIntosh SW. 2004a. Helioseismic mapping of the magnetic canopy in the solar chromosphere. Astrophys J Lett 613(2): L185-L188. https://doi. org/10.1086/424996.

Finsterle W, Jefferies SM, Cacciani AR, Rapex P, Giebink C, Knox A, Dimartino V. 2004b. Seismology of the solar atmosphere. Sol Phys 220(2): 317-331. https://doi.org/10.1023/B:SOLA. 0000031397.73790.7b.

Forte R, Jefferies SM, Berrilli F, Del Moro D, Fleck B, Giovannelli L, Murphy N, Pietropaolo E, Rodgers W. 2018. The MOTH II Doppler-magnetographs and data calibration pipeline. In: Space weather of the heliosphere: Processes and forecasts, Foullon C, Malandraki OE (Eds.), Vol. 335 of IAU Symposium, pp. 335-339.

Forte R, Berrilli F, Calchetti D, Del Moro D, Fleck B, et al. 2020. A data reduction pipeline for MOF-based synoptic telescopes. Journal of Space Weather and Space Climate, this issue, https://doi.org/10.1051/swsc/2020065.

Fuller N, Aboudarham J, Bentley RD. 2005. Filament recognition and image cleaning on Meudon $\mathrm{H} \alpha$ spectroheliograms. Sol Phys 227(1): 61-73. https://doi.org/10.1007/s11207-005-8364-1.

Giannattasio F, Berrilli F, Biferale L, Del Moro D, Sbragaglia M, Bellot Rubio L, Gošić M, Orozco Suárez D. 2014. Pair separation of magnetic elements in the quiet Sun. $A \& A$ 569: A121. https://doi. org/10.1051/0004-6361/201424380.

Giannattasio F, Consolini G, Berrilli F, Del Moro D. 2019. The complex nature of magnetic element transport in the quiet Sun: The Lévy-walk character. Astrophys $J$ 878(1): 33. https://doi.org/ 10.3847/1538-4357/ab1be2.

Giersch O.. 2013. GONG inter-site $\mathrm{H} \alpha$ flare comparison. In: Journal of Physics Conference Series, Vol. 440 of Journal of Physics Conference Series, 012006 p. https://doi.org/10.1088/1742-6596/ 440/1/012006.

Gosain S, Roth M, Hill F, Pevtsov A, Pillet VM, Thompson MJ. 2018. Design of a next generation synoptic solar observing network: Solar physics research integrated network group (SPRING). In: Ground-based and airborne instrumentation for astronomy VII, Vol. 10702, Evans CJ, Simard L, Takami H (Eds.), International Society for Optics and Photonics, SPIE, pp. 13551363. https://doi.org/10.1117/12.2306555.

Haberreiter M, Finsterle W, Jefferies SM. 2007. On the observation of traveling acoustic waves in the solar atmosphere using a magneto-optical filter. Astron Nachr 328(3): 211. https://doi.org/ 10.1002/asna.200610721.

Harvey JW, Hill F, Hubbard RP, Kennedy JR, Leibacher JW, et al. 1996. The Global Oscillation Network Group (GONG) Project. Science 272(5266): 1284-1286. https://doi.org/10.1126/ science.272.5266.1284. https://ui.adsabs.harvard.edu/abs/1996Sci... 272.1284H. Provided by the SAO/NASA Astrophysics Data System.

Hill F, Thompson MJ, Roth M. 2013. Workshop report: A new synoptic solar observing network. Space Weather 11(7): 392-393. https://doi.org/10.1002/swe.20068.

Iarlori M, Pietropaolo E, Rizi V, Aramo C, Valore L. 2019. The Raman LIDAR for the pre-production phase of Cherenkov 
Telecope Array. In: European Physical Journal Web of Conferences, Vol. 197 of European Physical Journal Web of Conferences, 02004 p. https://doi.org/10.1051/epjconf/201919702004.

Jefferies SM, Pomerantz MA, Duvall TL Jr, Harvey JW, Jaksha DB. 1988. Helioseismology from the south pole: Comparison of 1987 and 1981 results. In: Seismology of the Sun and Sun-like stars, Rolfe EJ (Ed.), Vol. 286 of ESA Special Publication, pp. 279-284.

Jefferies SM, Fleck B, Murphy N, Berrilli F. 2019. Observed local dispersion relations for magnetoacoustic-gravity waves in the Sun's atmosphere: Mapping the acoustic cutoff frequency. Astrophys J Lett 884(1): L8. https://doi.org/10.3847/2041-8213/ ab4719.

Jurčák J, Collados M, Leenaarts J, van Noort M, Schlichenmaier R. 2019. Recent advancements in the EST project. Adv Space Res 63 (4): 1389-1395. https://doi.org/10.1016/j.asr.2018.06.034.

Leenaarts J, Carlsson M, Rouppe van der Voort L. 2012. The formation of the $\mathrm{H} \alpha$ line in the solar chromosphere. Astrophys $J$ 749(2): 136. https://doi.org/10.1088/0004-637X/749/2/136.

Moretti PF, Cacciani A, Hanslmeier A, Messerotti M, Oliviero M, Otruba W, Severino G, Warmuth A. 2001. The source of the solar oscillations: Convective or magnetic? A\&A 372: 1038-1047. https://doi.org/10.1051/0004-6361:20010588.

Moretti PF, Cacciani A, Hanslmeier A, Messerotti M, Otruba W, Warmuth A. 2003. Full-disk magnetic oscillations in the solar photosphere. A\&A 403: 297-302. https://doi.org/10.1051/0004-6361: 20030366.

Moretti PF, Berrilli F, Bigazzi A, Jefferies SM, Murphy N, Roselli L, di Mauro MP. 2010. Future instrumentation for solar physics: A double channel MOF imager on board ASI Space Mission ADAHELI. Astrophys Space Sci 328(1-2): 313-318. https://doi. org/10.1007/s10509-009-0251-z.

Murphy N, Smith E, Rodgers W, Jefferies S. 2005. Chromospheric observations in the helium $1083 \mathrm{~nm}$ line - A new instrument. In: Solar wind 11/SOHO 16, Connecting Sun and Heliosphere, Fleck B, Zurbuchen TH, Lacoste H (Eds.), Vol. 592 of ESA Special Publication, 511 p.

Napoletano G, Forte R, Moro DD, Pietropaolo E, Giovannelli L, Berrilli F. 2018. A probabilistic approach to the drag-based model. J Space Weather Space Clim 8: A11. https://doi.org/10.1051/swsc/ 2018003.

Oliviero M, Severino G, Straus T. 1998. VAMOS: velocity and intensity data analysis and first results on I-V phase difference at low 1. Mem Soc Astron Italiana 69: 623.

Oliviero M, Severino G, Esposito G. 2010. Planning magneto-optical filters for the study of magnetic oscillations of the Sun. Astrophys Space Sci 328(1-2): 325-329. https://doi.org/10.1007/s10509010-0360-8.

Pesnell WD, Thompson BJ, Chamberlin PC. 2012. The solar dynamics observatory (SDO). Sol Phys 275(1-2): 3-15. https://doi.org/10.1007/s11207-011-9841-3.

Piazzesi R, Berrilli F, Del Moro D, Egidi A. 2012. Algorithm for real time flare detection. Mem Soc Astron Italiana Suppl 19: 109.
Plainaki C, Antonucci M, Bemporad A, Berrilli F, Bertucci B, et al. 2020. Current state and perspectives of Space Weather science in Italy. J Space Weather Space Clim 10: 6. https://doi.org/10.1051/ swsc/2020003.

Pötzi W, Veronig AM, Riegler G, Amerstorfer U, Pock T, Temmer M, Polanec W, Baumgartner DJ. 2015. Real-time flare detection in ground-based $\mathrm{H} \alpha$ imaging at Kanzelhöhe observatory. Sol Phys 290(3): 951-977. https://doi.org/10.1007/s11207-014-0640-5.

Pötzi W, Veronig AM, Temmer M. 2018. An event-based verification scheme for the real-time flare detection system at Kanzelhöhe observatory. Sol Phys 293(6): 94. https://doi.org/10.1007/s11207018-1312-7.

Rast M, Cauzzi G, Martinez Pillet V. 2019. The critical science plan for DKIST. Nuovo Cimento C Geophys Space Phys C 42(1): 7. https://doi.org/10.1393/ncc/i2019-19007-0.

Schrijver CJ. 2007. A characteristic magnetic field pattern associated with all major solar flares and its use in flare forecasting. Astrophys $J$ Lett 655(2): L117-L120. https://doi.org/10.1086/511857.

Severino G, Oliviero M, Landi Degl'Innocenti E. 2007. Simulation of magneto-optical filter transmission profiles. In: The physics of chromospheric plasmas. Heinzel P, Dorotovič I, Rutten RJ (Eds.), Vol. 368 of Astronomical Society of the Pacific Conference Series, $617 \mathrm{p}$.

Tomczyk S, Streander K, Card G, Elmore D, Hull H, Cacciani A. 1995. An instrument to observe low-degree solar oscillations. Sol Phys 159(1): 1-21. https://doi.org/10.1007/BF00733027.

Townson MJ, Kellerer A, Osborn J, Butterley T, Morris T, Wilson RW. 2015. Characterising daytime atmospheric conditions on $\mathrm{La}$ Palma. In: Journal of Physics Conference Series, Vol. 595 of Journal of Physics Conference Series, 012035 p. https://doi.org/ 10.1088/1742-6596/595/1/012035.

Vernazza JE, Avrett EH, Loeser R. 1981. Structure of the solar chromosphere. III. Models of the EUV brightness components of the quiet sun. Astrophys J Suppl Ser 45: 635-725. https://doi.org/ 10.1086/190731.

Veronig AM, Pötzi W. 2016. Ground-based observations of the solar sources of space weather. In: Coimbra solar physics meeting: Ground-based solar observations in the space instrumentation era, Dorotovic I, Fischer CE, Temmer M (Eds.), Vol. 504 of Astronomical Society of the Pacific Conference Series, 247 p.

Viavattene G, Calchetti D, Berrilli F, Del Moro D, Giovannelli L, Pietropaolo E, Oliviero M, Terranegra L. 2020. Optical design of the Tor vergata Synoptic Solar Telescope (TSST). In: Il Nuovo Ciment C (Ed.), Vol. 43 of Il Nuovo Cimento C, 120 p. https://doi. org/10.1393/ncc/i2020-20120-6.

Vogt E, Oliviero M, Severino G, Straus T. 1999. Calibration of VAMOS magnetic data. In: Magnetic fields and solar processes, Wilson A. (Ed.), Vol. 9 of ESA Special Publication, 405 p.

Wang Y, Cao H, Chen J, Zhang T, Yu S, Zheng H, Shen C, Zhang J, Wang S. 2010. Solar Limb Prominence Catcher and Tracker (SLIPCAT): An automated system and its preliminary statistical results. Astrophys J 717(2): 973-986. https://doi.org/10.1088/ 0004-637X/717/2/973.

Cite this article as: Giovannelli L, Berrilli F, Calchetti D, Del Moro D, Viavattene G, et al. 2020. The Tor Vergata Synoptic Solar Telescope (TSST): A robotic, compact facility for solar full disk imaging. J. Space Weather Space Clim. 10, 58. https://doi.org/10.1051/swsc/2020061. 